This is an open access article distributed under the terms of the Creative Commons BY-NC-ND Licence

\title{
Expression of genes encoding PIP aquaporins in tomato seeds exposed to blue radiation and mercury
}

\author{
J. BALARYNOVÁ and M. FELLNER*
}

Laboratory of Growth Regulators, Centre of the Region Haná for Biotechnological and Agricultural Research, Palacký University and Institute of Experimental Botany, Academy of Sciences of the Czech Republic, CZ-78371 Olomouc, Czech Republic

\begin{abstract}
Aquaporins control the specific transport of water and some other small molecules across membranes and are involved in various physiological processes. Plasma membrane aquaporins (PIPs) were shown to play an important role during tomato seed development. Therefore, we were interested in the participation of PIPs in seed germination and early seedling growth of wild type (WT) and the $7 B-1$ mutant, affected by blue light responses. We characterised the expression patterns of PIP-type aquaporin genes in these lines during different phases of seed germination and seedling growth after $\mathrm{HgCl}_{2}$ (an aquaporin blocker) treatment. Further, we investigated whether blue radiation (BL) was involved in the regulation of these processes. Our experiments showed that $7 B-1$ mutant seed germination and root elongation are less responsive to $\mathrm{HgCl}_{2}$ compared to WT. In both WT and $7 B-1$ mutant seeds, BL modulates the expression of PIP $1 ; 1$ (upregulation) and $P I P 1 ; 3$ (downregulation) aquaporin isoforms. The PIP1;3 gene is downregulated not only by BL but also by $\mathrm{HgCl}_{2}$ with a stronger effect in WT seeds. Thus, we show that BL can alter PIPS gene expression during tomato seed germination and seedling growth and that the $7 B-1$ mutation reduced the responsiveness to mercury blockage of aquaporins. Altogether, our data indicate that $P I P$ aquaporins participate in tomato seed germination and radicle elongation and that the $7 B-1$ mutation and BL have an impact on these processes.
\end{abstract}

Additional key words: 7B-1 mutant, seed germination, Solanum lycopersicum.

\section{Introduction}

Seed germination commences with the uptake of water by dry seed and terminates with the elongation of the embryonic axis (Bewley and Black 1994), while the visible sign of completed germination is the penetration of the structures surrounding the embryo by the radicle (Bewley 1997). Seed germination is driven by the uptake of water which occurs in three phases: germination is triggered by high water uptake during imbibition (phase I) followed by a period of limited water uptake (lag phase, phase II). Finally, water uptake increases in phase III during the elongation of the radicle and the whole embryonic axis (Bewley and Black 1994). Aquaporins control water movements and maintain water homeostasis in plant cells. They represent an alternative pathway to simple diffusion of water across membranes. Via both routes, the water molecules move passively in response to water potential gradients, but the water passage through aquaporins is faster than the simple diffusion (Steudle 1994). It is expected that aquaporins are not involved in the early process of imbibition, but they are possibly important during embryo growth (Willigen et al. 2006).

Tomato aquaporins are classified into five main groups (Chaumont et al. 2001, Danielson and Johanson 2008). From 47 aquaporin genes in the tomato genome,

Submitted 30 June 2017, last revision 19 June 2018, accepted 20 June 2018.

Abbreviations: BL - blue radiation; $\mathrm{C}_{\mathrm{T}}$ - threshold cycle; D - darkness; DAS - day after sowing; DTT - dithiothreitol; MS - the basal Murashige and Skoog medium; PIPs - plasma membrane intrinsic proteins; RT-qPCR - reverse transcription quantitative PCR; WT - wild-type.

Acknowledgments: The authors thank Véronique Bergougnoux for assistance with analyses of gene expression and Jan Humplík for valuable discussion about the manuscript. We further thank Jiři Danihlík for beneficial discussion about data and statistical analyses. We thank Renáta Plotzová and Věra Chytilová for technical assistance. We thank Vipen K. Sawhney for providing the seeds of $7 B-1$ mutant and Jan Nauš for measurements of photon flux density. The authors thank Peter Hedden (Rothamsted Research, UK) for proof-reading the manuscript.

* Corresponding author; e-mail: martin.fellner@upol.cz 
14 encoded plasma membrane intrinsic proteins (PIPs) (Reuscher et al. 2013). It was shown that PIPs play an important homeostatic role during seed development (Shiota et al. 2006) and they were proposed to be involved in rehydration during seed germination (Gao et al. 1999, Suga et al. 2001, Schuurmans et al. 2003).

A useful tool to study a contribution of PIPs in seed germination and early post-germination growth is treatment with mercuric chloride. Although a few mercury-resistant aquaporins have been described in plants (Daniels et al. 1994, Biela et al. 1999, Willigen et al. 2006), mercury compounds are traditionally used as blockers of aquaporins and of water transport (Maggio and Joly 1995, Daniels et al. 1996, Chaumont et al. 1998, Biela et al. 1999, Hukin et al. 2002, Willigen et al. 2006, Beaudette et al. 2007). Mercury derivatives act by oxidizing cysteine residues within the water pore and altering the conformation in other regions of the protein without affecting the integrity of the cell. The blockage of aquaporins by $\mathrm{HgCl}_{2}$ can be reversed by reducing agents such as dithiothreitol (DTT) (Preston et al. 1992).

The expression of aquaporins can be modulated by various environmental factors including irradiance (Chaumont and Tyerman 2014, Li et al. 2014), but there is no report of the regulation of aquaporin expression by blue radiation (BL) during seed germination. We demonstrated earlier that $\mathrm{BL}$ reduces tomato seed germination and that tomato $7 B-1$ mutant seeds are less responsive to BL-induced inhibition of seed germination (Piterková et al. 2012). Kaldenhoff et al. (1995, 1996)

\section{Materials and methods}

Plants and germination: Tomato (Solanum lycopersicum L.) cv. Rutgers (wild-type, WT) and the $7 B-1$ mutant impaired in various responses to $\mathrm{BL}$ were used in this study. The seeds were surface sterilized in $2.8 \%(\mathrm{~m} / \mathrm{v})$ sodium hypochlorite solution (Bochemia, Bohumín, Czech Republic) for $20 \mathrm{~min}$ and then rinsed with sterile distilled water. Afterwards, seeds were sown on basal Murashige and Skoog (1962; MS) medium supplemented with $0.7 \%(\mathrm{v} / \mathrm{v})$ agar in round Petri dishes ( $90 \mathrm{~mm}$ in diameter). Based on the experiment, the MS medium was supplemented with 0,20 , or $30 \mu \mathrm{M} \mathrm{HgCl}_{2}$, or $2 \mathrm{mM} \mathrm{DTT}$, or with a mixture of $30 \mu \mathrm{M} \mathrm{HgCl}_{2}$ and $2 \mathrm{mM}$ DTT. The dishes were placed vertically in growth chambers (Microclima 1000, Snijders Scientific, Tilburg, The Netherlands) and seeds were incubated at a temperature of $23{ }^{\circ} \mathrm{C}$ and air humidity of ?? \%, under continuous BL or in darkness. For dark conditions, plates were wrapped in aluminium foil. BL was provided by fluorescent tubes (TLD-36W/18-Blue, Philips, Einhoven, The Netherlands) giving maximum irradiance of $10 \mu \mathrm{mol} \mathrm{m} \mathrm{m}^{-2} \mathrm{~s}^{-1}$ at $\left.440 \mathrm{~nm}\right)$. The light spectrum was measured using a portable spectroradiometer (model LI-1800; Li-COR, Lincoln, NE, USA). Seed germination demonstrated that continuous BL can alter aquaporin gene expression in Arabidopsis cell cultures. However, information linking these observations to provide a mechanism for BL-induced inhibition of seed germination via aquaporins is still missing.

A spontaneous recessive single gene mutant $7 B-1$ was selected for its male sterility (Sawhney 1997). Seed germination tests show that $7 B-1$ is less responsive than the corresponding wild type (WT) to the inhibitory effects of osmotic stress specifically under BL (Fellner and Sawhney 2002). This indicates that BL can modulate the inhibitory effect of abiotic stresses on tomato seed germination. Other results suggest that the $7 B-1$ mutation impairs BL signalling pathways, possibly the phototropin pathway (Bergougnoux et al. 2012, Hlavinka et al. 2013), and it probably affects the downstream components of the light signalling pathways (Omidvar and Fellner 2015). Recently, a genetic characterization of the $7 B-1$ mutant was published and SlGLO2 gene was proposed as a candidate gene underlying the $7 B-1$ mutation (Pucci et al. 2017). However, the precise characterisation of the $7 B-1$ mutation is still in progress.

The aims of our work were: 1) to investigate the responsiveness of tomato seed germination and early radicle and root elongation to mercury treatment (an aquaporin blockage); 2) to characterise the expression of PIP genes during seed germination and early radicle elongation; 3) to analyse the putative $\mathrm{BL}$ control over these processes.

was recorded daily from the second to eighth day after sowing. The germination percentage [(germinated seeds/total number of seeds $) \times 100]$ was counted every day and the average was calculated. For each treatment at least six independent experiments per each time point were performed and thirty seeds per dish were sown for each treatment.

Tomato seeds were sown on the Petri dishes with MS medium supplemented with 0 or $30 \mu \mathrm{M} \mathrm{HgCl}_{2}$ and cultured in darkness or $\mathrm{BL}$ as described above. After specified times $(4,8,12,24,48,72$ and $96 \mathrm{~h}$ after sowing), non-germinated seeds were collected and excess water was removed by a filter paper. Subsequently, seeds were weighed, dried at $70{ }^{\circ} \mathrm{C}$ for $48 \mathrm{~h}$ and then weighed again. The water content was calculated as [(FM $\mathrm{DM}) / \mathrm{FM} \times 100]$, where $\mathrm{FM}$ is the fresh mass of imbibed seeds and DM is seed dry mass. The experiment was repeated twice.

Tomato seeds were sown on MS medium in square Petri dishes $(120 \times 120 \mathrm{~mm})$ and incubated in the growth chambers in darkness as described above. After $3 \mathrm{~d}$, germinated seeds were transferred onto dishes containing MS medium supplemented with $\mathrm{HgCl}_{2}(0-30 \mu \mathrm{M})$ and 
incubated in growth chambers in the dark or under continuous BL for $7 \mathrm{~d}$. Then, the seedling root length was measured with a ruler. For each $\mathrm{HgCl}_{2}$ concentration, at least three independent experiments with at least seven seedlings were performed.

RNA isolation, cDNA synthesis, and PCR: For analysis of PIP expression in different developmental stages, the first set of seeds was harvested $24 \mathrm{~h}$ after sowing (nonvisible radicle). The second set comprised germinated seeds collected after $72 \mathrm{~h}$ of cultivation (the radicle was $1-3 \mathrm{~mm}$ long). Finally, seeds with radicle up to $10 \mathrm{~mm}$ were harvested $96 \mathrm{~h}$ after sowing. To study the effect of $\mathrm{HgCl}_{2}$ on PIP expression, WT and $7 B-1$ seeds with radicle up to $10 \mathrm{~mm}$ were harvested after 96 -h cultivation in the dark or under BL. Seeds germinated under BL were harvested at BL, while seeds germinated in the dark were harvested under a green safelight. Dry WT and $7 B-1$ seeds were also extracted. All seeds were ground by a mortar and pestle to a fine powder under liquid nitrogen. Total RNA from the seeds was isolated using the RNeasy Plant Mini kit (Qiagen, Hilden, Germany) according to the manufacturer's protocol. Residual DNA was removed from samples by treatment with recombinant DNaseI (Takara, Tokyo, Japan) and recombinant RNase inhibitor (Takara) at $37{ }^{\circ} \mathrm{C}$ for $60 \mathrm{~min}$. DNaseI was inactivated by phenol/chloroform extraction according to the manufacturer's instructions. Following this, first-strand cDNA mixtures were prepared from $0.7 \mu \mathrm{g}$ of total RNA using PrimeScript ${ }^{\mathrm{TM}} 1^{\text {st }}$ strand cDNA synthesis kit (Takara).

To screen changes of PIP gene expression induced by $30 \mu \mathrm{M} \mathrm{HgCl}_{2}$ and the differences in the amount of $P I P$ transcripts in WT and $7 B-1$ mutant seeds, a reverse transcription (RT) semiquantitative (sq)PCR was used. Gene expression was analysed with primers specific for nine tomato PIP genes (Shiota et al. 2006). The reaction mixture $\left[0.1 \mathrm{~mm}^{3}\right.$ of GoTaq ${ }^{\circledR}$ DNA polymerase (Promega, Madison, USA), $4 \mathrm{~mm}^{3}$ of $5 \times$ colorless GoTaq ${ }^{\circledR}$ reaction buffer (Promega), $1 \mathrm{~mm}^{3}$ of each primer $(10 \mu \mathrm{M}), 2 \mathrm{~mm}^{3}$ of dNTP mixture $(1 \mathrm{mM}), 9.8$ $\mathrm{mm}^{3}$ of sterile RNase-free water and $1 \mathrm{~mm}^{3}$ of cDNA template] volume was $20 \mathrm{~mm}^{3}$. DNA was denatured at $94{ }^{\circ} \mathrm{C}$ for $3 \mathrm{~min}$, followed by 25 or 30 cycles of amplification $\left(94{ }^{\circ} \mathrm{C}\right.$ for $30 \mathrm{~s}, 53-60{ }^{\circ} \mathrm{C}$ for $30 \mathrm{~s}$ and $72{ }^{\circ} \mathrm{C}$ for $1 \mathrm{~min}$ ) and final extension at $72{ }^{\circ} \mathrm{C}$ for $5 \mathrm{~min}$. LeEF1 (Elongation Factor 1 $\alpha$ ) (Shiota et al. 2006) and GAPDH (glyceraldehyde-3-phosphate dehydrogenase; primer kindly provided by $\mathrm{V}$. Bergougnoux: F-AACCGGTGTCTTCACTGACAAGGA, R-CACCCA CAACAAACATGGGAGCAT) were used as references. The PCR products were separated on 1 or $1.5 \%(\mathrm{~m} / \mathrm{v})$ agarose gel with ethidium bromide staining. The bands were evaluated using ImageJ program (Collins 2007) and gene expression relative to both reference genes and to control sample. The experiments with $\mathrm{HgCl}_{2}$ were repeated four times, experiments with dry seeds three times.

To assess the expression of PIPS during different stages of seed germination and early post-germination and to study the effect of $30 \mu \mathrm{M} \mathrm{HgCl} 2$ on PIP1;1 and $P I P 1 ; 3$ gene expression, RT-qPCR was used. The gene expression was analysed using SYBR Premix Ex Taq ${ }^{\mathrm{TM}}$ (perfect real-time kit; Takara) using a Mx3000P ${ }^{\mathrm{TM}}$ thermal cycler (Agilent Technologies, Palo Alto, USA). The PCR reaction mixture was prepared according to the manufacturer's protocol, containing $12.5 \mathrm{~mm}^{3}$ of $S Y B R$ Premix Ex Taq ${ }^{\mathrm{TM}}, 0.5 \mathrm{~mm}^{3}$ of each primer $(10 \mu \mathrm{M})$, $0.5 \mathrm{~mm}^{3}$ of ROX Reference Dye II, $9 \mathrm{~mm}^{3}$ of sterile RNase-free water and $2 \mathrm{~mm}^{3}$ of cDNA template (diluted 1/50). The final reaction volume was $25 \mathrm{~mm}^{3}$. Each sample was measured in triplicate. The following PCR running conditions were: an initial denaturation at $95{ }^{\circ} \mathrm{C}$ for $30 \mathrm{~s}$, followed by 40 cycles of $95{ }^{\circ} \mathrm{C}$ for $5 \mathrm{~s}$, and $60{ }^{\circ} \mathrm{C}$ for $20 \mathrm{~s}$. Melt curves were analysed at the end of PCR reactions. PP2Acs (Protein phosphatase 2A catalytic subunit) (Lovdal and Lillo 2009) and TIP41-like (Dekkers et al. 2012) genes were used as references. The sequences of aquaporin primers are given in Balarynová et al. 2018. The specific primers for PIP1;1 gene (accession number BP887068) were F-ACAAGG ACTACAAAGAGCCAC, R- GATTTAGAAACGCCC ATGACG. The primers were designed using PrimerQuest ${ }^{\circledR}$ program (IDT, Coralville, USA) and their efficiencies were calculated from the slope of the dilution curve. The series of 50,100,500, and 1000-fold diluted cDNA was prepared and measured in triplicate. The relative expression of $P I P 1 ; 1$ and $P I P 1 ; 3$ genes under mercury treatment was quantified against the expression in untreated WT seeds in the dark using delta-delta $\mathrm{C}_{\mathrm{T}}$ method and the experiment was repeated four times. The expression of individual PIPS in seeds collected 24, 72, and $96 \mathrm{~h}$ after sowing was calculated using delta $\mathrm{C}_{\mathrm{T}}$ method (the expression was relative to reference genes) (Pfaffl 2001). For PIP1;1, PIP1;3, PIP1;4, and PIP2;2 genes, whose expressions were low, the relative expression was quantified also using delta-delta $\mathrm{C}_{\mathrm{T}}$ method (against both WT and reference genes). The experiment was repeated three times.

Statistical analysis: The STATISTICA 12 software (StatSoft, Tulsa, OK, USA) was used to perform all statistical analyses. The significant differences among the different $\mathrm{HgCl}_{2}$ treatments were assessed using KruskalWallis test with multiple comparisons at the 0.05 significance level. To compare the difference between WT and $7 B-1$ mutant root length in the dark versus BL, the Mann-Whitney $U$-test was used (significance level 0.05). Mann-Whitney $U$-test was also performed to test the differences in root length of WT and $7 B-1$ seedlings after various $\mathrm{HgCl}_{2}$ treatments. The results of germination assay are presented as box plots. The bottom and the top of the box are made by the first 
and third quartiles and the central tendency of the variable is shown as a median (the band inside the box, the square inside the box represents a mean). The whiskers indicate outliers (1.5 times interquartile range). The box plots were done using OriginPro (OriginLab, Northampton, MA, USA). For the gene expression analyses, the number of biological repeats was not sufficient to prove statistical significance. In those cases, the original values of relative expression calculated using delta- $\mathrm{C}_{\mathrm{T}}$ method or delta-delta $\mathrm{C}_{\mathrm{T}}$-method (Pfaffl 2001) are given in the Supplement to demonstrate that the gene expression of individual replicates show the same trends (Table 1 - 5 Suppl.). These values were used to calculate the geometrical means and SE.

\section{Results}

To study the interaction between aquaporins and $\mathrm{BL}$ during seed germination, WT and $7 B-1$ mutant seeds were germinated in vitro in the presence of $\mathrm{HgCl}_{2}$, a generally utilized inhibitor of aquaporins (Macey 1984). In the absence of $\mathrm{HgCl}_{2}$ (control), $87 \%$ of WT seeds germinated after $7 \mathrm{~d}$ in the dark (D-control) while WT seed germination was slightly, but not significantly, reduced to $82 \%$ in continuous $\mathrm{BL}$ (BL-control) (Fig $1 A$ ). Application of $20 \mu \mathrm{M} \mathrm{HgCl} \mathrm{Hg}_{2}$ reduced WT seed germination to 37 and $40 \%$ in the dark as well as under $\mathrm{BL}$, respectively, compared to the corresponding control and $30 \mu \mathrm{M} \mathrm{HgCl} 2$ inhibited WT seed germination to 7 and $3 \%$ in the dark and under BL, respectively. In the dark as well as under BL, inhibition of WT seed germination by $\mathrm{HgCl}_{2}$ was completely restored when the seeds were treated simultaneously with $2 \mathrm{mM}$ dithiothreitol (DTT) but DTT alone did not have any significant effect on seed germination. WT seed

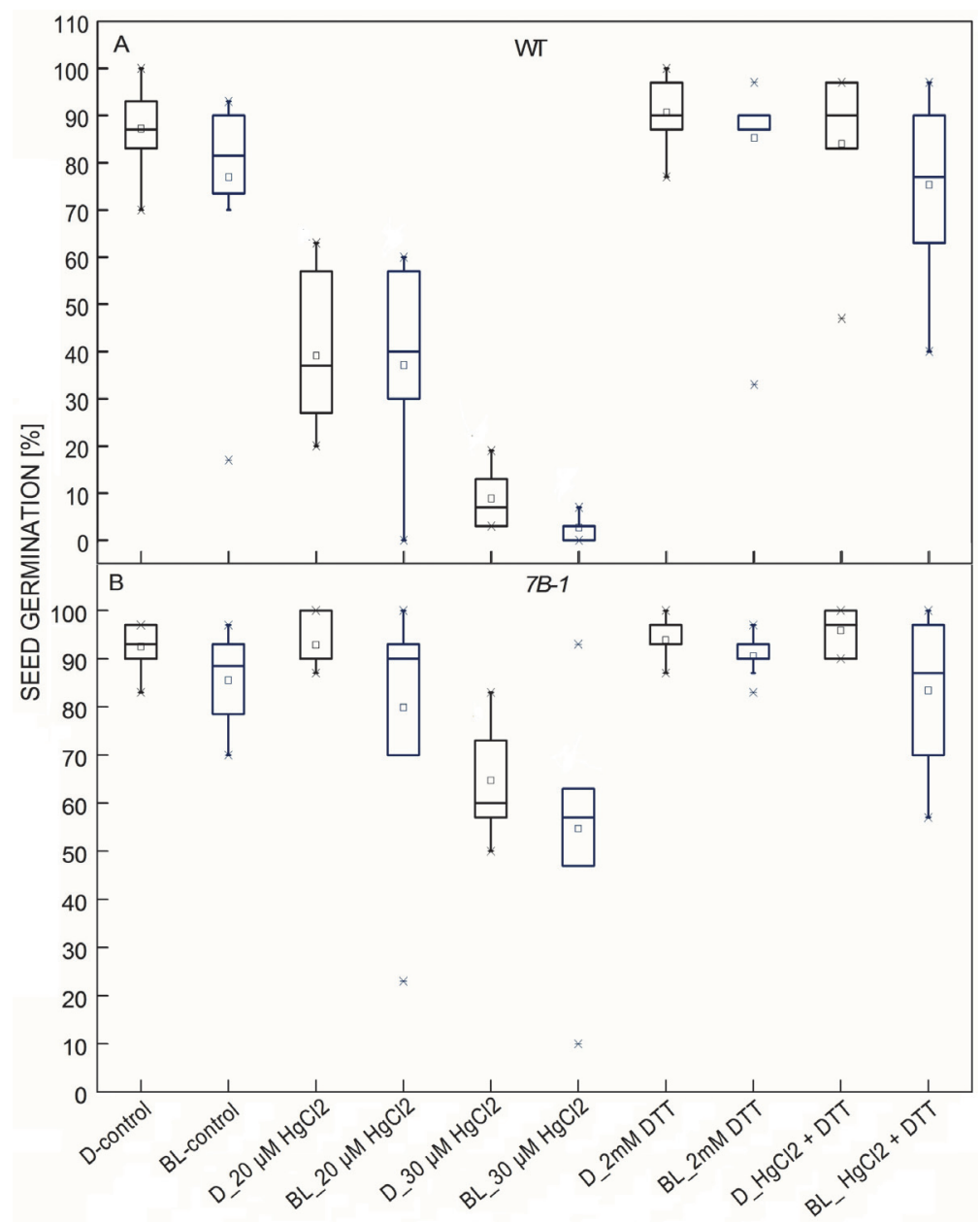

Fig. 1. Germination of tomato wild type (WT) $(A)$ and $7 B-1$ mutant $(B)$ seeds in the dark (D) or under blue light (BL) for $7 \mathrm{~d}$ as affected by $\mathrm{HgCl}_{2}$ and/or $2 \mathrm{mM}$ dithiothreitol (DTT). The boxes represent the first and third quartiles and a median value of seed germination (the band inside the box, the square inside the box represents a mean). The whiskers indicate outliers (1.5 times interquartile range), the small individual crosses showing outliers outside 1.5 times interquartile range. For each treatment, at least six independent experiments were done. Thirty seeds were sown on every dish. 
germination was also $90 \%$ on medium supplemented with DDT alone or with both $2 \mathrm{mM}$ DTT and $30 \mu \mathrm{M}$ $\mathrm{HgCl}_{2}$ in the dark while it reached $77 \%$ after the same treatment under BL (Fig $1 A)$.

Germination of $7 B-1$ mutant seeds (Fig $1 B$ ) reached 93 and $89 \%$ on control medium in the dark and under $\mathrm{BL}$, respectively. Germination of $7 B-1$ mutant seeds was not affected significantly by $20 \mu \mathrm{M} \mathrm{HgCl}_{2}$ in the dark and BL but $30 \mu \mathrm{M} \mathrm{HgCl}$ decreased $7 B-1$ seed germination to $60 \%$ in the dark and to $57 \%$ under BL compared to the corresponding control. Seed germination of $7 B-1$ mutant was also not affected by treatment with $2 \mathrm{mM}$ DTT alone (seed germination was $93 \%$ in the dark and $90 \%$ under $\mathrm{BL}$ ) and $97 \%$ of $7 B-1$ mutant seed germinated on medium with both $2 \mathrm{mM}$ DTT and $30 \mu \mathrm{M} \mathrm{HgCl}$ in the dark and $87 \%$ under BL.

The dynamics of seed germination in response to the aquaporin blocker were assessed by determining the rate of germination over 8 days after sowing (DAS) (Fig. 2). Mercury treatment markedly delayed WT seed germination. In the control medium and in the dark, average germination of WT and $7 B-1$ seeds reached the threshold of $25 \%$ before $3 \mathrm{~d}$ after sowing (DAS; Fig. $2 A, C)$. In the presence of $20 \mu \mathrm{M} \mathrm{HgCl}_{2}$, germination of WT seeds reached $25 \%$ by $4^{\text {th }}$ DAS. At $30 \mu \mathrm{M} \mathrm{HgCl}_{2}$, germination of WT seeds failed to reach $25 \%$. When $20 \mu \mathrm{M} \mathrm{HgCl} 2$ was applied, $7 B-1$ germination reached the threshold at approximately the $3^{\text {rd }}$ DAS in the dark, while $30 \mu \mathrm{M} \mathrm{HgCl}$ delayed mutant seed germination, reaching $25 \%$ at $4^{\text {th }}$ DAS. In the control medium and under BL, WT and $7 B-1$ mutant germination reached the $25 \%$ also by the $3^{\text {rd }}$ DAS (Fig. $2 B, D$ ). Treatment with $20 \mu \mathrm{M} \mathrm{HgCl}_{2}$ decreased the rate of WT seed germination under $\mathrm{BL}$ achieving the $25 \%$ threshold by $5^{\text {th }}$ DAS, whereas the effect of $30 \mu \mathrm{M} \mathrm{HgCl}_{2}$ was so pronounced that WT seed germination did not reach $25 \%$. Treatment with $20 \mu \mathrm{M}$ $\mathrm{HgCl}_{2}$ did not alter the kinetics of $7 B-1$ seed germination under $\mathrm{BL}$, although $30 \mu \mathrm{M} \mathrm{HgCl} 2$ markedly delayed $7 B-1$ seed germination under BL reaching the $25 \%$ threshold by $5^{\text {th }}$ DAS.

The higher resistance of $7 B-1$ seed germination to inhibition by mercury led us to test water uptake by dry WT and $7 B-1$ seeds in the absence or presence of $30 \mu \mathrm{M}$ $\mathrm{HgCl}_{2}$. In both genotypes, mercury had no effect on fresh mass increase during 96-h incubation of non-germinated seeds. As expected, BL did not affect the imbibition of water by dry WT and 7B-1 mutant seeds (Fig. 1 Suppl.).

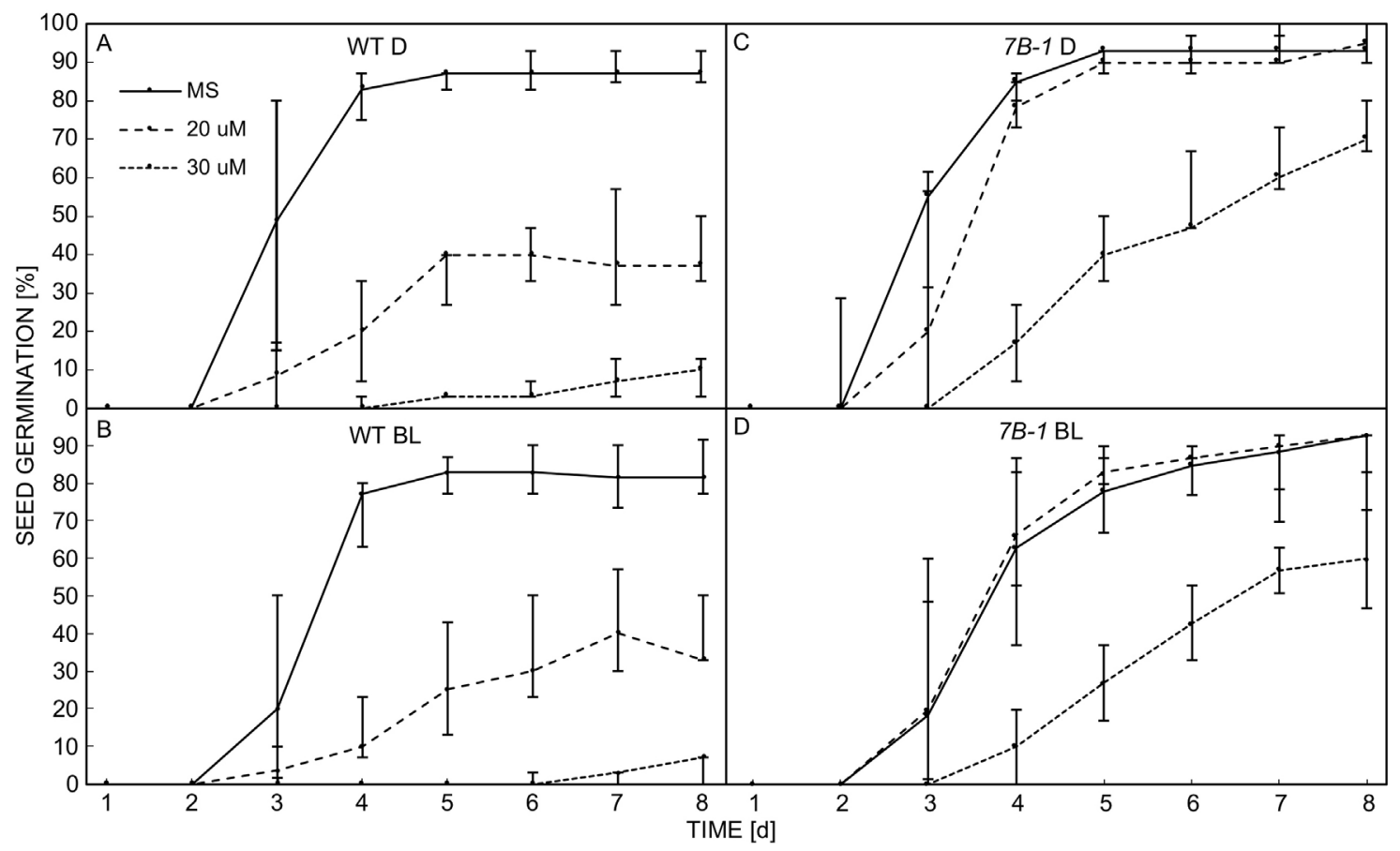

Fig. 2. The kinetics of WT $(A, B)$ and $7 B-1(C, D)$ seed germination on MS medium (control) and on MS supplemented with 20 or $30 \mu \mathrm{M} \mathrm{HgCl}_{2}$ during 8-d cultivation in the dark (D) or under blue light (BL). The data show medians and the error bars represent the first and third quartiles. For each treatment there were at least six independent experiments per each time point. Thirty seeds per each dish were sown for each treatment.

To test the sensitivity of developing roots to the aquaporin blocker $\mathrm{HgCl}_{2}$, root elongation of seedlings growing on different $\mathrm{HgCl}_{2}$ concentrations was studied. In the dark control, roots of 7-d-old WT seedlings were approximately $65 \mathrm{~mm}$ long. Root growth of WT seedlings was strongly inhibited by all applied concentrations of $\mathrm{HgCl}_{2}$ (Fig. $3 A$ ). $\mathrm{BL}$ stimulated significantly root growth in WT, whereas application of 
$\mathrm{HgCl}_{2}$ inhibited root growth counteracting the positive effect of $\mathrm{BL}$ (Fig. $3 \mathrm{~B}$ ). $\mathrm{HgCl}_{2}$ from $12 \mu \mathrm{M}$ concentration inhibited significantly the length of WT roots in the dark and BL. On control medium, roots in dark-grown $7 B-1$ seedlings (Fig. $3 A$ ) reached a similar length as WT roots but the inhibitory effect of $\mathrm{HgCl}_{2}$ on root elongation was markedly lower than in WT plants. In the dark at all tested $\mathrm{HgCl}_{2}$ concentrations, $7 B-1$ mutant roots were significantly longer than those of WT seedlings. BL stimulated root growth in $7 B-1$ plants (Fig. $3 B$ ) in a similar way as in WT plants but the WT seedlings had significantly longer roots than $7 B-1$ mutant seedlings growing on control MS medium. Responsiveness of the BL-incubated mutant roots to $\mathrm{HgCl}_{2}$ was significantly lower than in BL-incubated WT roots (Fig. 3B). Concentrations of $\mathrm{HgCl}_{2} \quad 12 \mu \mathrm{M}$ and higher inhibited significantly the length of $7 B-1$ roots in the dark, while under $\mathrm{BL}$, significant reduction in root length of $7 \mathrm{~B}-1$ control occurred at $15 \mu \mathrm{M} \quad \mathrm{HgCl}_{2}$ and higher concentrations.

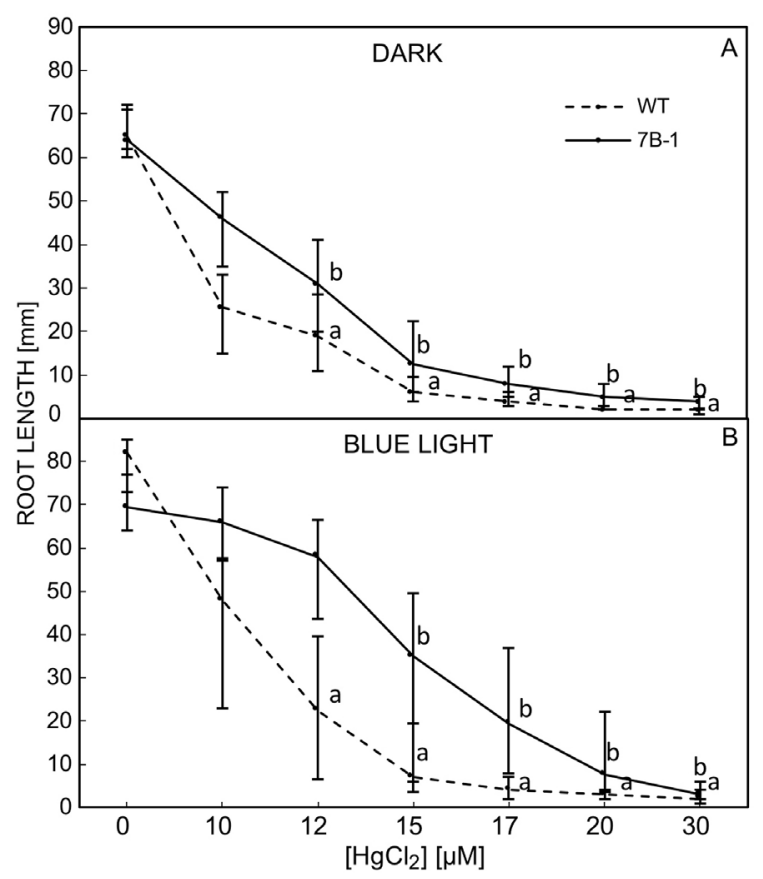

Fig. 3. The effect of increasing $\mathrm{HgCl}_{2}$ concentration on root length of 7-d-old WT and $7 B-1$ mutant seedlings growing in the dark $(A)$ or under blue light $(B)$. The results represent the medians and the error bars are the first and third quartiles. The significant difference among various treatments compared to MS medium without $\mathrm{HgCl}_{2}$ (control, mercury concentration 0) was tested using KruskalWallis test $(0.05<\mathrm{P})$, where a indicates a significant difference against WT control, b marked the significant difference compared to $7 B-1$ control. At least seven seedlings were measured per treatment and the experiment was repeated three times.

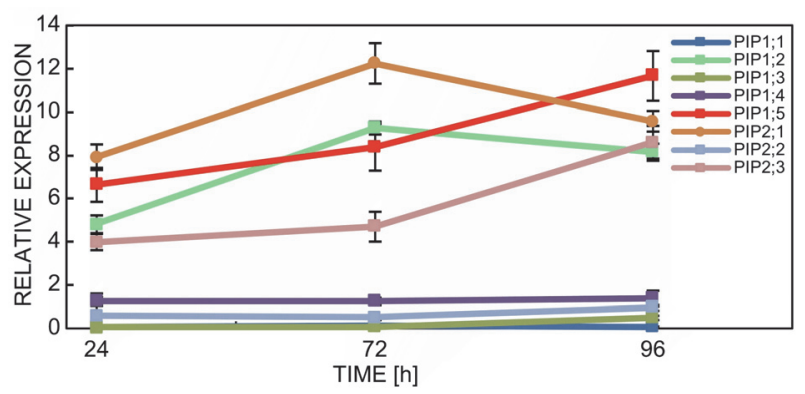

Fig. 4. The expression of PIPs during WT seed germination and early post-germination in the dark. RNA was isolated from seeds harvested 24, 72 (seeds with radicle $1-3 \mathrm{~mm}$ long), and $96 \mathrm{~h}$ (seeds with radicle $1-10 \mathrm{~mm}$ long) after seed sowing on MS medium. The expression was analysed by RT-qPCR. The expression was calculated relative to the expression of references genes $P P 2 A c s$ and TIP4llike. The values represent geometrical means and SEs of three independent experiments.

For a better understanding of the possible involvement of aquaporins in seed germination and early radicle elongation, we investigated the expression profiles of nine PIP genes (PIP1;1 to PIP1;5 and PIP 2;1 to
PIP2;4) (Shiota et al. 2006) in dry, imbibed and germinated seeds and in seeds cultured in the absence or presence of $\mathrm{HgCl}_{2}$.

In dry WT and $7 B-1$ seeds, transcripts of $P I P 1 ; 3$ and 
PIP2;4 genes were not detected. Expression of all other aquaporin genes tested showed a trend of lower expression in $7 B-1$ than in WT seeds (Fig. 2 Suppl.).

To investigate a possible involvement of the PIP aquaporins in BL-regulated seed germination and early radicle growth, we studied expression of the PIP genes in seeds incubated in the dark or in BL for 24, 72, and $96 \mathrm{~h}$ (see Materials and Methods). The only gene that was not expressed during seed germination and early postgermination was PIP2;4. Furthermore, we found that all other genes tested showed a trend of the highest expression after radicle protrusion (Fig 4, Fig. 4 Suppl.). The expression of each gene tended to be the lowest $24 \mathrm{~h}$ after sowing (during water imbibition) and then it increased during germination and the early seedling growth (72 and $96 \mathrm{~h}$ after sowing). This trend was the same for both WT and $7 B-1$ mutant seeds in the dark as well as under BL (Fig. 3 Suppl.). Expression of PIP1;2 and $P I P 2 ; 1$ genes tended to be highest at $72 \mathrm{~h}$ after seed sowing, whereas $P I P 1 ; 5$ and $P I P 2 ; 3$ expression peaked one day later ( $96 \mathrm{~h}$ after seed sowing, Fig. 4).

Interestingly, expression of $P I P 1 ; 1$ and $P I P 1 ; 3$ was modulated by BL (Fig. 5). In both genotypes, the expression of $P I P 1 ; 1$ gene showed a marked stimulation by $\mathrm{BL}$ in germinated seeds harvested $96 \mathrm{~h}$ after sowing (Fig. 5 and Fig. 4 Suppl.). A slight increase in PIP1;1 gene expression under BL was also observed in WT seeds harvested at $72 \mathrm{~h}$, but not at $24 \mathrm{~h}$ after sowing. Promotion of $P I P 1 ; 1$ gene expression by $\mathrm{BL}$ was not observed in $7 B-1$ mutant seeds collected after 24 and $72 \mathrm{~h}$ (Fig. 4 Suppl.). The expression of PIP1;3 was regulated by BL in the opposite way showing slight downregulation by BL in germinated seeds of both genotypes at $96 \mathrm{~h}$ after sowing (Fig. 5). However, no effect of BL on PIP1;3 gene expression was observed in WT and $7 B-1$ seeds collected 24 or $72 \mathrm{~h}$ after sowing (Fig. 4 Suppl.).

To investigate resistance of $7 B-1$ seed germination to $\mathrm{HgCl}_{2}$ at gene expression level, we studied expression of the PIP genes in germinated seeds (radicle length up to $1 \mathrm{~cm}$ ) incubated for $96 \mathrm{~h}$ in the absence or presence of $30 \mu \mathrm{M} \mathrm{HgCl}_{2}$ in the dark or under BL. From the nine PIP genes tested, only the expression of $P I P 1 ; 3$ was affected by $\mathrm{HgCl}_{2}$ (Fig. 5). The PIP 1;3 expression was reduced by $\mathrm{HgCl}_{2}$ in both WT and $7 B-1$ mutant seeds in the dark as well as under $\mathrm{BL}$ but the inhibition of $P I P 1 ; 3$ was less pronounced in $7 B-1$ than in WT seeds. $\mathrm{HgCl}_{2}$ had no effect on expression of PIP1;1 (Fig. 5), PIP2;1 and PIP2;3 (Fig. 5 Suppl.). However, the expressions of $P I P 1 ; 2, P I P 1 ; 4, P I P 1 ; 5$, and PIP2;2 genes were elevated in WT seeds incubated in the presence of $\mathrm{HgCl}_{2}$ under BL (Fig. 5 Suppl.).

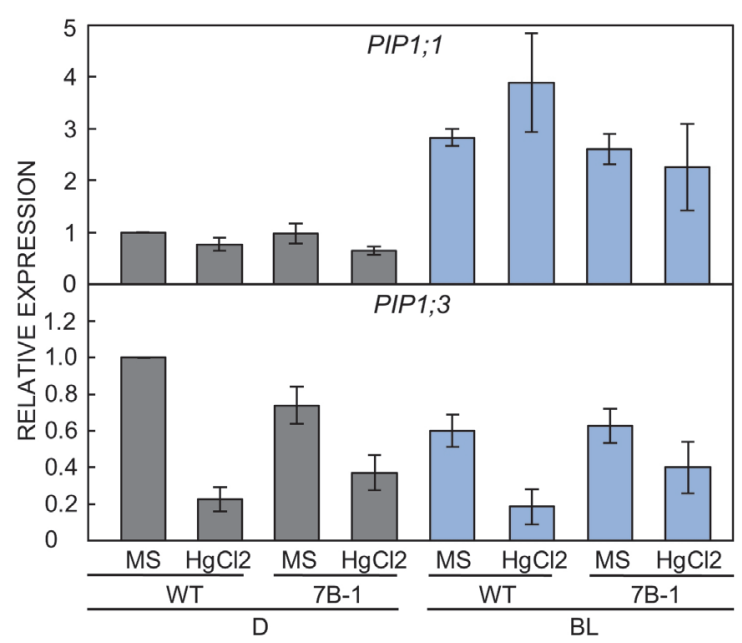

Fig. 5. The relative expression of $P I P 1 ; 1$ and $P I P 1 ; 3$ genes in WT and $7 B-1$ mutant seeds after 96-h cultivation on MS medium (control) or on MS medium with $30 \mu \mathrm{M} \mathrm{HgCl}_{2}$ in the dark (D) or under blue light (BL). The expression was measured by RT-qPCR. The relative expression was quantified against the expression in WT seeds in the dark and values represent the geometrical mean \pm SE of four experiments.

\section{Discussion}

Water uptake and its distribution to individual cells are essential physiological processes that are also the key factors during seed germination. The main role in plantwater relations is attributed to aquaporins which form the water selective pores in the membranes (Chaumont and Tyerman 2014). In this study, we investigated the prospective involvement of PIP aquaporins in tomato seed germination and early seedling growth at both the physiological and transcription levels using the standard water channel blocker $\mathrm{HgCl}_{2}$. We used the tomato $7 B-1$ mutant as its seed germination shows reduced responsiveness to the inhibition imposed by $\mathrm{BL}$ and 
various abiotic stresses compared to its corresponding WT (Fellner and Sawhney 2001, 2002).

Our experiments showed that $\mathrm{HgCl}_{2}$ suppressed significantly a final percentage of WT seed germination, indicating the importance of mercury-sensitive aquaporins. Moreover, seed germination in the $7 B-1$ mutant was less affected by $\mathrm{HgCl}_{2}$ than the WT. Application of $\mathrm{HgCl}_{2}$ caused not only reduction of the final portion of germinated seeds, but also delayed germination (radicle emergence), similarly as it has been reported in Arabidopsis (Willigen et al. 2006). Delayed radicle emergence was even more inhibited by $\mathrm{HgCl}_{2}$ under BL and this effect of BL was observed in the WT as well as in the less BL-responsive $7 B-1$ mutant. Our results demonstrated the lower responsiveness of $7 B-1$ mutant seed germination to aquaporin blockage compare to the WT. This suggests that the $7 B-1$ mutation may cause an increase in aquaporin amount or enhance their activity. However, we think that aquaporins were unlikely to be regulated by $7 B-1$ at the transcription level.

To prevent the potential toxic effects of mercury, we used relatively low concentrations of $\mathrm{HgCl}_{2}$, which were reported not to have any poisonous effects on seed germination (Willigen et al. 2006). Besides, we were able to restore seed germination by application of a reducing agent DTT. The reversibility of mercury inhibition of seed germination by DTT showed that $\mathrm{HgCl}_{2}$ did not cause irreversible disrupting of cell integrity (Willigen et al. 2006).

Our experiments imply that mercury-sensitive aquaporins are involved in tomato seed germination. Thereupon, we focused on water uptake during seed imbibition and on the possible effects of $\mathrm{HgCl}_{2}$ and $\mathrm{BL}$ on this process. The rate of water absorption is not altered by mercury during imbibition of Arabidopsis (Willigen et al. 2006) and pea seeds (Veselova and Veselovsky 2006). Our experiments confirmed that also in tomato, aquaporins do not participate in water uptake during seed imbibition. It was originally suggested that PIPS could play a role in rehydration during seed germination because their expression is elevated in germinating seeds in comparison to dry seed (Gao et al. 1999, Suga et al. 2001, Schuurmans et al. 2003). Further support for this notion is that commencement of seed germination includes extensive changes in water content which can be provided sufficiently only by rapid uptake through aquaporins. However, according to Veselova and Veselovsky (2006) aquaporins are closed during imbibition to prevent rapid water uptake by dry seeds which could damage them. In fact, it is likely that during imbibition water enters the seeds just by diffusion.

The role of PIPS in tomato seed development has been shown recently. Shiota et al. (2006) reported the expression of seven PIPS (PIP1;1, PIP1;2, PIP1;3, PIP1;4, PIP1;5, PIP2;1 and PIP2;2) in developing tomato seeds and they found that the amount of aquaporin proteins increases and maintains high in mature seeds.
Thus, we focused on the characterization of the expression patterns of $P I P \mathrm{~s}$ during tomato WT and $7 B-1$ mutant seed germination and early post-germination. We found that transcripts of the tested PIPs, apart from PIP1;3 and PIP2;4, preexisted in the dry seeds. Surprisingly, dry seeds of $7 B-1$ contained lower amounts of PIP transcripts than did dry WT seeds despite the fact that $7 B-1$ seeds coped better than WT seed with mercuryinhibition. The fact that $P I P 1 ; 3$ was not expressed in dry seeds, but it was detected in imbibed and germinating seeds, primarily during radicle elongation, indicates the importance of PIP 1;3 in this process. The absence of PIP2;4 expression in dry, imbibed, or germinating seeds suggest that it is probably not involved in seed germination.

The amount of PIP transcripts tended to increase together with radicle protrusion. Thus, a role for PIPs in radicle elongation could be anticipated. This is supported by the facts that $\mathrm{HgCl}_{2}$ decreased seed germination, but did not affect water imbibition of dry WT and $7 B-1$ seeds, which is consistent with a role for PIPs during radicle growth, but not during water imbibition. Besides, the expression of the studied aquaporin genes was highest in the seeds with a visible radicle. Indeed, cell elongation requires continuous uptake of water to maintain pressure potential (Chaumont and Tyerman 2014). The elongation of radicle cells is generally accepted to be sufficient for the completion of radicle protrusion, while cell division is not essential (Barroco et al. 2005), and a role for PIPs in tissue elongation has been suggested several times (Maurel et al. 2002, Fricke and Chaumont 2007, Liu et al. 2008).

Various trends in expression patterns were observed for the tested PIP genes suggesting their different roles in various phases of seed germination and early postgermination. PIP1;2, PIP1;5, PIP $2 ; 1$ and PIP $2 ; 3$ transcripts were prevalent, whereas $P I P 1 ; 1, P I P 1 ; 3$, $P I P 1 ; 4$, and PIP2;2 transcripts were much less abundant in germinating seeds. Although the seed germination assays demonstrated lower responsiveness of $7 B-1$ mutant seed germination to aquaporin blockage compared to WT, no fundamental difference in PIP transcripts accumulation was found between WT and mutant seeds in both the dark and under BL. Furthermore, not even mercury treatment $\left(30 \mu \mathrm{M} \mathrm{HgCl}_{2}\right)$ had a substantial effect on PIP gene expression, with only one exception. The amount of $P I P 1 ; 3$ transcripts was downregulated by mercury (Fig. 5). Moreover, PIP1;3 expression showed a moderate downregulation also by $\mathrm{BL}$ during radicle elongation. The same trend was found also in $7 B-1$ seeds, although the effect of mercury seemed to be more obvious in WT than in $7 B-1$ seeds. The relevance of effect of BL on PIP1;3 expression will be studied in detail using various $\mathrm{BL}$ intensities to confirm its impact on PIP 1;3 gene in tomato. It would be interesting to investigate further the protein expression of individual PIPs to find out whether they differ in WT and $7 B-1$ seeds. 
Specifically, $\mathrm{HgCl}_{2}$ enhances slightly the expression of PIP1;2, PIP1;4, PIP1;5 and PIP2;2 genes in WT seeds under BL. It is possible that mercury-induced blockage of aquaporins together with $\mathrm{BL}$ and this required increased transcription of these genes to compensate for the aquaporin blockage in order to sustain the appropriate root elongation. $7 B-1$ mutant seeds were not so greatly affected by the treatment with mercury, so there was no need to increase aquaporin gene transcription.

Alteration of aquaporin gene expression by BL was reported by Kaldenhoff et al. (1995, 1996), who found out that BL induces the transcription of aquaporin genes AthH2 and AthH3 in Arabidopsis cell cultures. However, a comprehensive study of $\mathrm{BL}$ effects on aquaporin function during seed germination and early seedling growth was still missing. In our study, PIP 1;3 gene expression was affected by BL (see above). Besides, BL stimulated the expression of $P I P 1 ; 1$ in both WT and $7 B-1$ seeds. The facts that the $P I P 1 ; 1$ expression is highest in seeds with elongating radicles and that $P I P 1 ; 1$ transcript accumulation is stimulated by $\mathrm{BL}$ could suggest the involvement of this gene in BL-induced growth of WT and $7 B-1$ roots as discussed below.

To investigate the physiological role of aquaporins in root elongation, we studied the effect of $\mathrm{HgCl}_{2}$ on root length of WT and $7 B-1$ mutant seedlings. We showed that root elongation of WT and $7 B-1$ mutant seedlings was suppressed by $\mathrm{HgCl}_{2}$, supporting the expectation that aquaporins are involved in this process (Hukin et al. 2002, Javot and Maurel 2002). However, $7 B-1$ mutant roots were less responsive to mercury inhibition than those of WT. In both genotypes, root growth was stimulated significantly by BL compared to dark-growth

\section{References}

Balarynová, J., Danihlík, J., Fellner, M.: Changes in plasma membrane aquaporin gene expression under osmotic stress and blue light in tomato. - Acta Physiol. Plant. 40: 27, 2018.

Barroco, R.M., Van Poucke, K., Bergervoet, J.H.W., De Veylder, L., Groot, S.P.C., Inze, D., Engler, G.: The role of the cell cycle machinery in resumption of postembryonic development. - Plant Physiol. 137: 127-140, 2005.

Beaudette, P.C., Chlup, M., Yee, J., Emery, R.N.: Relationships of root conductivity and aquaporin gene expression in Pisum sativum: diurnal patterns and the response to $\mathrm{HgCl}_{2}$ and $\mathrm{ABA}$. - J. exp. Bot. 58: 1291-1300, 2007.

Bergougnoux, V., Hlaváčková, V., Plotzová, R., Novák, O., Fellner, M.: The $7 B-1$ mutation in tomato (Solanum lycopersicum L.) confers a blue light-specific lower sensitivity to coronatine, a toxin produced by Pseudomonas syringae pv. tomato. - J. exp. Bot. 60: 1219-1230, 2009.

Bergougnoux, V., Zalabák, D., Jandová, M., Novák, O., WieseKlinkenberg, A., Fellner, M.: Effect of blue light on endogenous isopentenyladenine and endoreduplication during photomorphogenesis and de-etiolation of tomato (Solanum lycopersicum L.) seedlings. - PLoS ONE 7: e45255, 2012. seedlings. The effect of light on root elongation is well known in many plant species and the possible pathway through which roots perceive light to promote their elongation is described for Arabidopsis (Dyachok et al. 2011). $7 B-1$ seedling growing on MS under BL conditions had shorter roots than WT seedling growing under the same conditions. This clearly demonstrates that the $7 B-1$ defect in BL signalling pathways results in lower responses to $\mathrm{BL}$ stimuli. On the contrary, in the presence of $\mathrm{HgCl}_{2}, 7 \mathrm{~B}-1$ roots were always longer that those of WT seedlings under BL indicating the lower responsiveness of $7 B-1$ root elongation to mercury treatment.

In summary, expression analyses of PIP genes indicate that, apart from PIP2;4, all tested PIPS participate in tomato seed germination and early radicle elongation. PIPs seemed to be involved in radicle elongation during seed germination with no indication of role in rapid water imbibition at the commencement of seed germination. We showed that the BL-induced expression of PIP1;1 correlated with BL-induced stimulation of root elongation, indicating that $P I P 1 ; 1$ could be involved in root elongation under BL. Furthermore, the relationship between the lower responsiveness of $7 B-1$ mutant seeds to mercury-induced inhibition of aquaporins and lower sensitivity of $P I P 1 ; 3$ gene expression to $\mathrm{HgCl}_{2}$ in $7 B-1$ seeds suggested a role of $P I P 1 ; 3$ in tomato radicle elongation. Thus, we showed that BL can alter PIP gene expression (PIP1;1 and $P I P 1 ; 3)$ during tomato seed germination and early postgermination and that the $7 B-1$ mutation (primarily considered to affect BL sensing) causes a lower responsiveness to mercury blockage of aquaporins.

Bewley, J.D.: Seed germination and dormancy. - Plant Cell 9: 1055-1066, 1997.

Bewley, J.D., Black, M.: Seeds. Physiology of Development and Germination. - Plenum Press, New York 1994.

Biela, A., Grote, K., Otto, B., Hoth, S., Hedrich, R., Kaldenhoff, R.: The Nicotiana tabacum plasma membrane aquaporin NtAQP1 is mercury-insensitive and permeable for glycerol. Plant J. 18: 565-570, 1999.

Chaumont, F., Barrieu, F., Wojcik, E., Chrispeels, M.J., Jung, R.: Aquaporins constitute a large and highly divergent protein family in maize. - Plant Physiol. 125: 1206-1215, 2001.

Chaumont, F., Tyerman, S.D.: Aquaporins: highly regulated channels controlling plant water relations. - Plant Physiol. 164: 1600-1618, 2014.

Chaumont, F., Barrieu, F., Herman, E.M., Chrispeels, M.J.: Characterization of a maize tonoplast aquaporin expressed in zones of cell division and elongation. - Plant Physiol. 117: 1143-1152, 1998

Collins, T.J.: ImageJ for microscopy. - Biotechniques 43: 25-30, 2007.

Daniels, M.J., Mirkov, T.E., Chrispeels, M.J: The plasma 
membrane of Arabidopsis thaliana contains a mercuryinsensitive aquaporin that is homologue of the tonoplast water channel protein TIP. - Plant Physiol. 106: 1325-1333, 1994.

Daniels, M.J., Chaumont, F., Mirkov, T.E., Chrispeels, M.J.: Characterization of a new vacuolar membrane aquaporin sensitive to mercury at a unique site. - Plant Cell 8: 587-599, 1996.

Danielson, J.A., Johanson, U.: Unexpected complexity of the aquaporin gene family in the moss Physcomitrella patens. BMC Plant Biol. 8: 45, 2008.

Dekkers, B.J.W., Willems, L., Bassel, G.W., Van BolderenVeldkamp, R.P., Ligterink, W., Hilhorst, H.W.M., Bentsink, L.: Identification of reference genes for RT-qPCR expression analysis in Arabidopsis and tomato seeds. - Plant Cell Physiol. 53: 28-37, 2012.

Dyachok, J., Zhu, L., Liao, F., He, J., Huq, E., Blancaflor, E.B.: SCAR mediates light-induced root elongation in Arabidopsis through photoreceptors and proteasomes. - Plant Cell 23: 3610-3626, 2011.

Fellner, M., Sawhney, V.K.: Seed germination in a tomato malesterile mutant is resistant to osmotic, salt and low-temperature stresses. - Theor. appl. Genet. 102: 215-221, 2001.

Fellner, M., Sawhney, V.K.: The $7 B-1$ mutant in tomato shows blue-light-specific resistance to osmotic stress and abscisic acid. - Planta 214: 675-682, 2002.

Fricke, W., Chaumont, F.: Solute and water relations of growing plant cells. - In: Verbelen, J.P., Vissenberg, K. (ed.): The Expanding Cell. Pp. 7-31. Springer-Verlag, Berlin Heidelberg 2007.

Gao, Y.P., Young, L., Bonham-Smith, P., Gusta, L.V.: Characterization and expression of plasma and tonoplast membrane aquaporins in primed seed of Brassica napus during germination under stress conditions. - Plant mol. Biol. 40: 635-644, 1999.

Hlavinka, J., Nauš, J., Fellner, M.: Spontaneous mutation $7 B-1$ in tomato impairs blue light-induced stomatal opening. - Plant Sci. 209: 75-80, 2013.

Hukin, D., Doering-Saad, C., Thomas, C.R., Pritchard, J.: Sensitivity of cell hydraulic conductivity to mercury is coincident with symplastic isolation and expression of plasmalemma aquaporin genes in growing maize roots. Planta 215: 1047-1056, 2002.

Javot, H., Maurel, C.: The role of aquaporins in root water uptake. - Ann. Bot. 90: 301-313, 2002.

Kaldenhoff, R., Kölling, A., Meyers, J., Karmann, U., Ruppel, G., Richter, G.: The blue light-responsive AthH2 gene of Arabidopsis thaliana is primarily expressed in expanding as well as in differentiating cells and encodes a putative channel protein of the plasmalemma. - Plant J. 7: 87-95, 1995.

Kaldenhoff, R., Kölling, A., Richter, G.: Regulation of the Arabidopsis thaliana aquaporin gene AthH2 (PIP1b).- J. Photochem. Photobiol. B Biol. 36: 351-354, 1996.

Li, G., Santoni, V., Maurel, C.: Plant aquaporins: roles in plant physiology. - Biochim. biophys. Acta 1840: 1574-1582, 2014.

Liu, D.Q., Tu, L.L., Wang, L., Li, Y.J., Zhu, L.F., Zhang, X.L.: Characterization and expression of plasma and tonoplast membrane aquaporins in elongating cotton fibers. - Plant Cell Rep. 27: 1385-1394, 2008.

Lovdal, T., Lillo, C.: Reference gene selection for quantitative real-time PCR normalization in tomato subjected to nitrogen, cold, and light stress. - Anal. Biochem. 237: 238-242, 2009.

Macey, R.I.: Transport of water and urea in red blood cells. Amer. J. Physiol. cell. Physiol. 246: C195-C203, 1984.

Maggio. A., Joly, R.J.: Effects of mercuric chloride on the hydraulic conductivity of tomato root systems (evidence for a channel-mediated water pathway). - Plant Physiol. 109: 331$335,1995$.

Maurel, C., Javot, H., Lauvergeat, V., Gerbeau, P., Tournaire, C., Santoni, V., Heyes, J.: Molecular physiology of aquaporins in plants. - Int. Rev. Cytol. 215: 105-148, 2002.

Murashige, T., Skoog, F.: A revised medium for rapid growth and bioassays with tobacco tissue cultures. - Physiol. Plant. 15: 473-497, 1962.

Omidvar, V., Fellner, M.: DNA methylation and transcriptomic changes in response to different lights and stresses in $7 B-1$ male-sterile tomato. - PLoS ONE 10: e0121864, 2015.

Pfaffl, M.W.: A new mathematical model for relative quantification in real-time RT-PCR. - Nucl. Acids Res. 29: e45-e45, 2001.

Piterková, J., Luhová, L., Hofman, J., Turečková, V., Novák, O., Petřivalský, M., Fellner, M.: Nitric oxide is involved in lightspecific responses of tomato during germination under normal and osmotic stress conditions. - Ann. Bot. 110: 767-776, 2012.

Preston, G.M., Carroll, T.P., Guggino, W.B., Agre, P.: Appearance of water channels in Xenopus oocytes expressing red cell CHIP28 protein. - Science 256: 385-387, 1992.

Pucci, A., Picarella, M.E., Mazzucato, A.: Phenotypic, genetic and molecular characterization of $7 B-1$, a conditional malesterile mutant in tomato. - Theor. appl. Genet. 130: 23612374, 2017.

Reuscher, S., Akiyama, M., Mori, C., Aoki, K., Shibata, D., Shiratake, K.: Genome-wide identification and expression analysis of aquaporins in tomato. - PLoS ONE 8: e79052, 2013.

Sawhney, V.K.: Genic male sterility. - In: Shivanna, K.R., Sawhney, V.K. (ed.): Pollen Biotechnology for Crop Production and Improvement. Pp 183-198. Cambridge University Press, Cambridge 1997.

Schuurmans, J.A., Van Dongen, J.T., Rutjens, B.P., Boonman, A., Pieterse, C.M., Borstlap, A.C.: Members of the aquaporin family in the developing pea seed coat include representatives of the PIP, TIP and NIP subfamilies. - Plant mol. Biol. 53: 633-645, 2003.

Shiota, H., Sudoh, T., Tanaka, I.: Expression analysis of genes encoding plasma membrane aquaporins during seed and fruit development in tomato. - Plant Sci. 171: 277-285, 2006.

Steudle, E.: Water transport across roots. - Plant Soil 167: 79-90, 1994.

Suga, S., Imagawa, S., Maeshima, M.: Specifity of the accumulation of mRNAs and proteins of the plasma membrane and tonoplast aquaporins in radish organs. - Planta 212: 294-304, 2001.

Veselova, T.V., Veselovsky, V.A.: Possible involvement of aquaporins in water uptake by pea seeds differing in quality. Russ. J. Plant. Physiol. 53: 96-101, 2006.

Willigen, C.V., Postaire, O., Tournaire-Roux, C., Boursiac, Y., Maurel, C.: Expression and inhibition of aquaporins in germinating Arabidopsis seeds. - Plant Cell Physiol. 47: 1241-1250, 2006. 